Editorial

\title{
New Perspectives Offered by Nuclear Medicine for the Imaging and Therapy of Multiple Myeloma
}

\author{
Charles Mesguich, Paolo Zanotti-Fregonara, Elif Hindié ${ }^{凶}$ \\ Service de Médecine Nucléaire, CHU de Bordeaux, LabEx TRAIL, Université de Bordeaux, France \\ $\triangle$ Corresponding author: Pr Elif Hindié, MD, PhD, Service de Médecine Nucléaire, Hôpital Haut-Lévêque, CHU de Bordeaux, 33604 Pessac, France. Tel: \\ +(33)557656838; Fax: +(33)557656839. e-mail: elif.hindie@chu-bordeaux.fr
}

(c) Ivyspring International Publisher. Reproduction is permitted for personal, noncommercial use, provided that the article is in whole, unmodified, and properly cited. See http://ivyspring.com/terms for terms and conditions.

Published: 2016.01.01

\begin{abstract}
The management of multiple myeloma has fundamentally changed over the years and imaging techniques able to match the therapeutic advances are now much needed. Although many patients now achieve complete response after first-line treatment, relapse is common. Therefore, it would be important to improve the initial prognostic stratification and to detect minimal residual disease after treatment. ${ }^{18} \mathrm{~F}$-FDG-PET/CT is a useful imaging tool which has a high prognostic value at baseline evaluation and can effectively differentiate active from inactive lesions during induction treatment or after autologous stem-cell transplantation. In combination with biological data, it improves the prediction of relapse. Other PET tracers may soon enter clinical practice and overcome some of the limitations of ${ }^{18} \mathrm{~F}$-FDG, such as the low sensitivity in detecting early bone marrow infiltration. Excellent results with "C-Methionine are reported by Lapa and colleagues in this issue of the Journal. "C-Methionine uptake reflects the increased protein synthesis of malignant plasmocytes and correlates well with bone marrow infiltration. Other promising PET ligands include lipid tracers, such as " $\mathrm{C}$-Choline or ${ }^{11} \mathrm{C}$-acetate, and some peptide tracers, such as ${ }^{68} \mathrm{Ga}$-Pentixafor, that targets CXCR4 (chemokine receptor-4), which is often expressed with high density by myeloma cells.

Malignant plasma cells are radiosensitive and thus potentially amenable to systemic radionuclide therapy. Indeed, excellent preclinical results were obtained with radioimmunotherapy targeting CD38. Also, preliminary clinical results with peptides targeting CXCR4 (e.g. ${ }^{177}$ Lu- or ${ }^{90} \mathrm{Y}$-Pentixather) are encouraging. Multiple myeloma may represent a renewal of the already strong partnership between hematologists and nuclear medicine physicians.
\end{abstract}

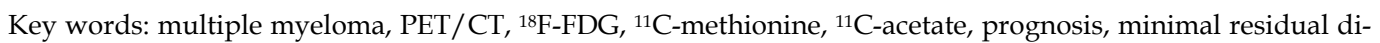
sease, radionuclide therapy, ${ }^{177} \mathrm{Lu}, \mathrm{CXCR} 4, \mathrm{CD} 38$

Nuclear medicine has substantially influenced the management of hematological patients. In many subtypes of lymphomas, ${ }^{18} \mathrm{~F}-\mathrm{FDG}-\mathrm{PET} / \mathrm{CT}$ improved initial staging, refined the definition of complete remission, and opened new perspectives for a tailored treatment based on early metabolic response (i.e. "interim PET") (1). Targeted radionuclide therapy, using anti-CD20 antibodies labeled with either ${ }^{131}$ I or ${ }^{90} \mathrm{Y}$, was successfully used to treat patients with relapsed or refractory non-Hodgkin lymphomas and, more recently, for consolidation of remission $(2,3)$. However, nuclear medicine has traditionally played a limited role in the management of multiple myeloma.

Multiple myeloma (MM) represents about 15\% of hematologic cancers. The median age at diagnosis is 69 years (4). MM is characterized by the proliferation of clonal plasma cells in the bone marrow, which causes an increased secretion of monoclonal immunoglobulins. These immunoglobulins are detectable in the serum or urine. Signs of organ damage include 
lytic bone lesions, renal impairment, hypercalcemia, and anemia. The median survival has increased from 3 to 6 years in the past two decades (4), mainly as a result of increased use of autologous stem cell transplantation and the introduction of immunomodulatory drugs (e.g. thalidomide, lenalidomide, pomalidomide) and proteasome inhibitors (e.g., bortezomib, carfilzomib).

Treatment of patients who are eligible for transplantation usually consists of two-drug (e.g. lenalidomide-dexamethasone or bortezomibdexamethasone) or three-drug induction regimen, followed by high dose melphalan and autologous stem-cell transplantation (4). Various combinations of active drugs can be used in patients not eligible for transplantation (e.g., melphalan, dexamethasone and bortezomib) (4). Thanks to these therapeutic strategies, many patients now achieve complete response, as defined by conventional serological and morphologic techniques (5). Unfortunately, the majority of patients still relapse. There is thus growing interest in improving the prognostic stratification at staging, as well as in improving the detection of minimal residual disease, which can be present inside or outside the bone marrow. Functional imaging might be one of the most promising tools to achieve these improvements. Indeed, early studies suggested that ${ }^{18} \mathrm{FDG}$ imaging at baseline and post-treatment may offer important prognostic information (6).

Staging of patients with MM in the revised International Staging System (R-ISS) is based only on biological parameters (serum $\beta 2$-microglobulin, albumin, and LDH levels) and the presence or absence of high-risk chromosomal abnormalities (7). Regarding diagnostic imaging, planar radiographies of the skeleton have been traditionally used as the standard screening technique, despite some known limitations such as low sensitivity and specificity and the inability to detect extraosseous lesions (8). It is now accepted that the diagnosis of myeloma bone disease can be made with whole-body low-dose CT, MRI or ${ }^{18}$ F-FDG-PET/CT (9).

Compared to other radiographic methods, MRI has higher sensitivity for early detection of marrow infiltration in the axial skeleton. It is the gold standard imaging technique for evaluating painful lesions and for distinguishing malignant versus benign (e.g. osteoporotic) vertebral fractures. Moreover, MRI can detect spinal cord or nerve compression and the presence of masses in the soft tissues (10).

However, the strengths of MRI should not obscure those of $18 \mathrm{~F}-\mathrm{FDG}-\mathrm{PET} / \mathrm{CT}$. At baseline, the presence of more than three ${ }^{18} \mathrm{~F}-\mathrm{FDG}$-avid bone lesions, the presence of extra-medullary disease, and the intensity of ${ }^{18} \mathrm{~F}-\mathrm{FDG}$ uptake have better prognostic value for overall survival than the parameters provided by MRI $(11,12)$. ${ }^{18} \mathrm{~F}-\mathrm{FDG}-\mathrm{PET} / \mathrm{CT}$ is the best imaging tool for distinguishing between active and inactive disease after therapy, and it allows for earlier and more specific evaluation of therapeutic efficacy compared to MRI (13). ${ }^{18} \mathrm{~F}-\mathrm{FDG}-\mathrm{PET} / \mathrm{CT}$ provides prognostic information during induction treatment $(11,14)$ or after autologous stem-cell transplantation (12). In combination with biological data, it improves the prediction of relapse $(15,16)$.

Correctly identifying minimal residual disease would be of paramount importance to predict the outcome of patients in apparent complete remission and to better manage the individual risk (e.g. choosing whether to use maintenance therapy or consolidation). The excellent results recently obtained by treating relapsed/refractory MM with anti-CD38 or anti-SLAMF7 monoclonal antibodies $(17,18)$ suggest that these treatments might soon be used earlier, for example for consolidation. Minimal residual disease can be detected in the bone marrow with immunophenotypic techniques based on multiparameter flow cytometry, or with PCR-based molecular techniques $(5,19)$. Patchy bone marrow infiltration, and extramedullary involvement, may however generate false negative results (19). ${ }^{18} \mathrm{~F}-\mathrm{FDG}-\mathrm{PET} / \mathrm{CT}$ can be an excellent complementary tool for detecting focal sites of activity. Indeed, ${ }^{18}$ F-FDG-PET/CT is highly sensitive for extra-medullary localizations $(6,20,21)$, and for detecting residual focal bone or bone marrow lesions $(11,12)$. However, a baseline study is important for assessing residual sites of uptake after therapy (11), as is the case in lymphomas $(1,22)$.

Limitations of ${ }^{18} \mathrm{~F}-\mathrm{FDG}-\mathrm{PET} / \mathrm{CT}$ include a lack of sensitivity for detecting diffuse bone marrow involvement (in this case MRI remains the gold standard) and low-density plasmocyte infiltration. Also, physiological ${ }^{18} \mathrm{~F}-\mathrm{FDG}$ uptake in the brain reduces the sensitivity for small skull lesions (23).

The prospective study by Lapa and colleagues in this issue of Theranostics presents an interesting option to overcome some of the limitations of ${ }^{18} \mathrm{~F}-\mathrm{FDG}$ (24). The authors performed a head-to-head comparison of ${ }^{11} \mathrm{C}$-Methionine and ${ }^{18} \mathrm{~F}-\mathrm{FDG}-\mathrm{PET} / \mathrm{CT}$ scans in 43 patients referred for staging or re-staging of MM (24). ${ }^{11} \mathrm{C}$-Methionine was better than ${ }^{18} \mathrm{~F}-\mathrm{FDG}$ at detecting both intra- and extramedullary lesions. ${ }^{11} \mathrm{C}$-Methionine found more focal lesions in 28 out of 43 patients $(65.1 \%$; $<<0.001)$, whereas in the other 15 patients both tracers yielded comparable results. The degree of bone marrow infiltration strongly correlated $(\mathrm{r}=0.9)$ with ${ }^{11} \mathrm{C}-M e t h i o n i n e ~ u p t a k e$ at the site of iliac crest biopsy, which was performed in 31 patients.

${ }^{11} \mathrm{C}$-Methionine has been used for imaging brain tumors such as gliomas, as well as for detecting par- 
athyroid lesions (25). The uptake of ${ }^{11} \mathrm{C}$-Methionine is correlated with L-type amino acid transporter 1 (LAT1) (26). Interestingly, LAT1 expression has been suggested as a prognostic marker in MM (27). ${ }^{11} \mathrm{C}$-Methionine uptake in MM probably reflects increased protein and immunoglobulin synthesis (28). In a pilot study by Dankerl and colleagues (29), MM patients with diffuse bone marrow involvement displayed significantly higher uptake than control patients (patients investigated for hyperparathyroidism).

The study of Lapa and colleagues shows impressive uptake of ${ }^{11} \mathrm{C}$-Methionine in $\mathrm{MM}$ lesions and substantially confirms other studies which found a higher sensitivity of ${ }^{11} \mathrm{C}$-Methionine compared to ${ }^{18}$ F-FDG $(30,31)$.

Some questions are however still unanswered:

- What is the prognosis of lesions displaying high ${ }^{11} \mathrm{C}-$ Methionine and low ${ }^{18} \mathrm{~F}-\mathrm{FDG}$ uptake and how do these lesions respond to current treatments?

- To what extent can the high physiological uptake of ${ }^{11} \mathrm{C}$-Methionine in the liver mask liver lesions? Although less frequent than other sites of extra-medullary disease (e.g., skin, soft tissue and lymph nodes) at initial presentation, the liver is more commonly involved during relapse (21).

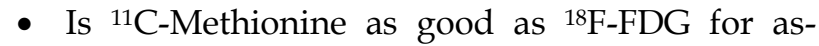
sessing response to treatments? Preliminary data in xenografted mice suggest that ${ }^{11} \mathrm{C}$-Methionine may be better than ${ }^{18}$ F-FDG as an early marker for response to proteasome inhibitors (32).

- MRI is recommended for the workup of patients with solitary bone plasmacytoma and those with smoldering "asymptomatic" myeloma $(9,10)$. Preliminary reports suggest that ${ }^{18} \mathrm{~F}-\mathrm{FDG}-\mathrm{PET} /$ CT is useful also in these patients $(33,34)$. Can ${ }^{11} \mathrm{C}-$ Methionine offer higher sensitivity?

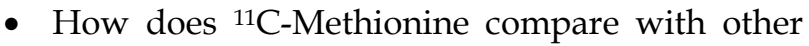
metabolic tracers, such as ${ }^{11} \mathrm{C}$-Choline (35) or ${ }^{11} \mathrm{C}$-acetate $(36,37)$ ? These tracers also are more sensitive than ${ }^{18} \mathrm{~F}-\mathrm{FDG}$ for detecting MM lesions (35-37).

Interestingly, some new peptides may specifically image MM lesions. ${ }^{68} \mathrm{Ga}$-Pentixafor is a peptide with high affinity for CXCR4 (chemokine receptor-4), which is often expressed with high density by MM cells (38). A recent study in 14 patients with advanced MM showed that ${ }^{68} \mathrm{Ga}$-Pentixafor had an excellent tumor-to-background ratio, although not all lesions were visualized, and ${ }^{18} \mathrm{~F}-\mathrm{FDG}-\mathrm{PET} / \mathrm{CT}$ revealed additional information in some patients (39). Interestingly, these peptides can be labeled also with $ß$-emitters and thus be used for targeted radiotherapy if all MM lesions in a patient show high receptor ex- pression. Indeed, preliminary results with ${ }^{177} \mathrm{Lu}-$ or ${ }^{90} Y$-Pentixather are encouraging (40).

The excellent results obtained by targeting CD38 with cold antibodies in patients with relapsed/refractory MM (17) raise the hope that an even stronger response might be obtained by conjugating CD38 antibodies with radioisotopes, as previously demonstrated with radiolabeled anti-CD20 antibodies in lymphoma (2). In one study, CD38-pretargeting/ ${ }^{90}$ Y-DOTA-biotin was very effective at eradicating subcutaneous plasmocytoma xenografts (41). Similarly, promising preclinical results were obtained with ${ }^{213}$ Bi-anti-CD38 (42). Many other receptors, such as CD138 (43), SLAMF7, CD40, CD54, IL-6 receptor, and CD74 are highly expressed in MM cells and they might be targeted for radioimmunotherapy.

In summary, the management of MM has fundamentally changed in the past two decades and imaging techniques able to match the therapeutic advances are much needed.

${ }^{18} \mathrm{~F}-\mathrm{FDG}$-PET/CT is an excellent tool that deserves wider clinical use. Other tracers may soon enter clinical practice. PET-CT imaging can be used in MM patients with renal impairment without safety concerns from contrast agents. PET-MRI would allow both MR and metabolic imaging within a single examination (44).

The effectiveness of external beam radiotherapy for treating plasmocytoma shows that malignant plasma cells are highly radiosensitive. Thus, MM patients may be good candidate for targeted radionuclide therapy, because this approach would deliver a high dose to disseminated tumors while relatively sparing the healthy tissues. In conclusion, MM may represent a renewal of the already strong partnership between hematologists and nuclear medicine physicians.

\section{References}

1. Cheson BD, Fisher RI, Barrington SF, et al. Recommendations for initial evaluation, staging, and response assessment of Hodgkin and non-Hodgkin lymphoma: the Lugano classification. J Clin Oncol. 2014;32:3059-68.

2. Witzig TE, Gordon LI, Cabanillas F, et al. Randomized controlled trial of yttrium-90-labeled ibritumomab tiuxetan radioimmunotherapy versus rituximab immunotherapy for patients with relapsed or refractory low-grade, follicular, or transformed B-cell non-Hodgkin's lymphoma. J Clin Oncol. 2002;20:2453-63.

3. Morschhauser F, Radford J, Van Hoof A, et al. Phase III trial of consolidation therapy with yttrium-90-ibritumomab tiuxetan compared with no additional therapy after first remission in advanced follicular lymphoma. J Clin Oncol. 2008;26:5156-64.

4. Röllig C, Knop S, Bornhäuser M. Multiple myeloma. Lancet. 2015;385:2197-208.

5. Rajkumar SV, Harousseau JL, Durie B, et al. Consensus recommendations for the uniform reporting of clinical trials: report of the International Myeloma Workshop Consensus Panel 1. Blood. 2011;117:4691-5.

6. Durie BG, Waxman AD, D'Agnolo A, Williams CM. Whole-body (18)F-FDG PET identifies high-risk myeloma. J Nucl Med. 2002;43:1457-63.

7. Palumbo A, Avet-Loiseau H, Oliva S, et al. Revised International Staging System for Multiple Myeloma: A Report From International Myeloma Working Group. J Clin Oncol. 2015;33:2863-9.

8. Dimopoulos M, Terpos E, Comenzo RL, et al. International myeloma working group consensus statement and guidelines regarding the current role of im- 
aging techniques in the diagnosis and monitoring of multiple Myeloma. Leukemia. 2009;23:1545-56.

9. Rajkumar SV, Dimopoulos MA, Palumbo A, et al. International Myeloma Working Group updated criteria for the diagnosis of multiple myeloma. Lancet Oncol. 2014;15:e538-48.

10. Dimopoulos MA, Hillengass J, Usmani S, et al. Role of magnetic resonance imaging in the management of patients with multiple myeloma: a consensus statement. J Clin Oncol. 2015;33:657-64.

11. Bartel TB, Haessler J, Brown TL, et al. F18-fluorodeoxyglucose positron emission tomography in the context of other imaging techniques and prognostic factors in multiple myeloma. Blood. 2009;114:2068-76

12. Zamagni E, Patriarca F, Nanni C, et al. Prognostic relevance of 18-F FDG $\mathrm{PET} / \mathrm{CT}$ in newly diagnosed multiple myeloma patients treated with up-front autologous transplantation. Blood. 2011;118:5989-95.

13. Derlin T, Peldschus K, Münster S, et al. Comparative diagnostic performance of ${ }^{18} \mathrm{~F}$-FDG PET/CT versus whole-body MRI for determination of remission status in multiple myeloma after stem cell transplantation. Eur Radiol. 2013;23:570-8.

14. Usmani SZ, Mitchell A, Waheed S, et al. Prognostic implications of serial 18-fluoro-deoxyglucose emission tomography in multiple myeloma treated with total therapy 3. Blood. 2013;121:1819-23.

15. Elliott BM, Peti S, Osman K, et al. Combining FDG-PET/CT with laboratory data yields superior results for prediction of relapse in multiple myeloma. Eur J Haematol. 2011;86:289-98.

16. Zamagni E, Nanni C, Mancuso K, et al. PET/CT Improves the Definition of Complete Response and Allows to Detect Otherwise Unidentifiable Skeletal Progression in Multiple Myeloma. Clin Cancer Res. 2015;21:4384-90.

17. Lokhorst HM, Plesner T, Laubach JP, et al. Targeting CD38 with Daratumumab Monotherapy in Multiple Myeloma. N Engl J Med. 2015;373:1207-19.

18. Lonial S, Dimopoulos M, Palumbo A, et al. Elotuzumab Therapy for Relapsed or Refractory Multiple Myeloma. N Engl J Med. 2015 Aug 13;373(7):621-31.

19. Paiva B, Puig N, García-Sanz R, et al. Is this the time to introduce minimal residual disease in multiple myeloma clinical practice? Clin Cancer Res. 2015;21:2001-8.

20. Bladé J, Fernández de Larrea C, Rosiñol L, Cibeira MT, Jiménez R, Powles R. Soft-tissue plasmacytomas in multiple myeloma: incidence, mechanisms of extramedullary spread, and treatment approach. J Clin Oncol. 2011;29:3805-12.

21. Usmani SZ, Heuck C, Mitchell A, et al. Extramedullary disease portends poor prognosis in multiple myeloma and is over-represented in high-risk disease even in the era of novel agents. Haematologica. 2012;97:1761-7.

22. Moulin-Romsee G, Hindié E, Cuenca X, et al. (18)F-FDG PET/CT bone/bone marrow findings in Hodgkin's lymphoma may circumvent the use of bone marrow trephine biopsy at diagnosis staging. Eur J Nucl Med Mol Imaging. 2010;37:1095-105.

23. Mesguich C, Fardanesh R, Tanenbaum L, Chari A, Jagannath S, Kostakoglu L. State of the art imaging of multiple myeloma: comparative review of FDG PET/CT imaging in various clinical settings. Eur J Radiol. 2014;83:2203-23.

24. Lapa C, Knop S, Schreder M, et al. ${ }^{11}$ C-Methionine-PET in Multiple Myeloma: Correlation with Clinical Parameters and Bone Marrow Involvement. Theranostics. 2016; 6(2): 254-261

25. Hindié $\mathrm{E}$, Zanotti-Fregonara $\mathrm{P}$, Tabarin $\mathrm{A}$, et al. The role of radionuclide imaging in the surgical management of primary hyperparathyroidism. J Nucl Med. 2015;56:737-44.

26. Okubo S, Zhen HN, Kawai N, Nishiyama Y, Haba R, Tamiya T. Correlation of L-methyl-11C-methionine (MET) uptake with L-type amino acid transporter 1 in human gliomas. J Neurooncol. 2010;99:217-25.

27. Isoda A, Kaira K, Iwashina M, et al. Expression of L-type amino acid transporter 1 (LAT1) as a prognostic and therapeutic indicator in multiple myeloma. Cancer Sci. 2014;105:1496-502.

28. Hammerton K, Cooper DA, Duckett M, Penny R. Biosynthesis of immunoglobulin in human immunoproliferative diseases. I. Kinetics of synthesis and secretion of immunoglobulin and protein by bone marrow cells in myeloma. J Immunol. 1978;121:409-17.

29. Dankerl A, Liebisch P, Glatting G, et al. Multiple Myeloma: Molecular Imaging with 11C-Methionine PET/CT--Initial Experience. Radiology. 2007;242:498-508.

30. Nakamoto $Y$, Kurihara $K$, Nishizawa $M$, et al. Clinical value of ${ }^{11} \mathrm{C}$-methionine $\mathrm{PET} / \mathrm{CT}$ in patients with plasma cell malignancy: comparison with ${ }^{8} \mathrm{~F}$-FDG PET/CT. Eur J Nucl Med Mol Imaging. 2013;40:708-15.

31. Okasaki $M$, Kubota $K$, Minamimoto $R$, et al. Comparison of (11)C-4'-thiothymidine, (11)C-methionine, and (18)F-FDG PET/CT for the detection of active lesions of multiple myeloma. Ann Nucl Med. 2015;29:224-32.

32. Lückerath K, Lapa C, Albert C, et al. 11C-Methionine-PET: a novel and sensitive tool for monitoring of early response to treatment in multiple myeloma. Oncotarget. 2015;6:8418-29.

33. Fouquet G, Guidez S, Herbaux C, et al. Impact of initial FDG-PET/CT and serum-free light chain on transformation of conventionally defined solitary plasmacytoma to multiple myeloma. Clin Cancer Res. 2014;20:3254-60.

34. Zamagni E, Nanni C, Gay F, et al. 18F-FDG PET/CT FOCAL, but not osteolytic, lesions predict the progression of smoldering myeloma to active disease. Leukemia. 2015 Oct 22. doi: 10.1038/leu.2015.291. [Epub ahead of print].

35. Nanni C, Zamagni E, Cavo M, et al. 11C-choline vs. 18F-FDG PET/CT in assessing bone involvement in patients with multiple myeloma. World J Surg Oncol. 2007;5:68.
36. Lin $\mathrm{C}$, Ho CL, $\mathrm{Ng} \mathrm{SH}$, et al. (11)C-acetate as a new biomarker for PET/CT in patients with multiple myeloma: initial staging and postinduction response assessment. Eur J Nucl Med Mol Imaging. 2014;41:41-9.

37. Ho CL, Chen S, Leung YL, et al. 11C-acetate PET/CT for metabolic characterization of multiple myeloma: a comparative study with 18F-FDG PET/CT. J Nucl Med. 2014;55:749-52.

38. Wester HJ, Keller U, Schottelius M, et al. Disclosing the CXCR4 expression in lymphoproliferative diseases by targeted molecular imaging. Theranostics. 2015;5:618-30.

39. Philipp-Abbrederis K, Herrmann K, Knop S, et al. In vivo molecular imaging of chemokine receptor CXCR4 expression in patients with advanced multiple myeloma. EMBO Mol Med. 2015;7:477-87.

40. Herrmann K, Schottelius M, Lapa C, et al. First-in-man experience of CXCR4-directed endoradiotherapy with 177Lu- and 90Y-labelled pentixather in advanced stage multiple myeloma with extensive intra- and extramedullary disease. J Nucl Med. 2015 Nov 12. pii: jnumed.115.167361. [Epub ahead of print]

41. Green DJ, Orgun NN, Jones JC, et al. A preclinical model of CD38-pretargeted radioimmunotherapy for plasma cell malignancies. Cancer Res. 2014;74:1179-89.

42. Teiluf K, Seidl C, Blechert B, et al. a-Radioimmunotherapy with ${ }^{213} \mathrm{Bi}$-anti-CD38 immunoconjugates is effective in a mouse model of human multiple myeloma. Oncotarget. 2015;6:4692-703.

43. Chérel M, Gouard S, Gaschet J, et al. 213Bi radioimmunotherapy with an anti-mCD138 monoclonal antibody in a murine model of multiple myeloma. J Nucl Med. 2013;54:1597-604.

44. Drzezga A, Souvatzoglou M, Eiber M, et al. First clinical experience with integrated whole-body PET/MR: comparison to PET/CT in patients with oncologic diagnoses. J Nucl Med. 2012;53:845-55. 